\title{
nature chemical biology
}

\section{Walking the walk}

U niversities and research institutes have been instrumental in the creation of scientific disciplines, as they nurture scholars whose contributions open new areas of scientific inquiry. They also serve as educational incubators for the next generation of scientists in these emerging fields. Recently, academic institutions around the world have recognized the growing importance of scholarship at the chemistry-biology interface and have sought to attract the most talented chemical biologists. However, recruiting innovative researchers and inspiring teachers is only the first step. Academic departments, universities and other institutions need to adopt a more active role. By highlighting some of the career challenges faced by chemical biologists, we hope to initiate a discussion about the ways scientists and administrators can better support the growth of chemical biology.

The lack of consistent standards for evaluating chemical biologists is a challenge for the advancement of chemical biology. First, because the discipline is a recent addition to most universities, there are few chemical biologists to serve as benchmarks for success. Second, chemical biologists are frequently delocalized across academic departments. Although most self-identified chemical biologists are in chemistry departments, it is becoming increasingly common to find these researchers in biology, engineering or medical-school departments. These academic units have significant cultural differences that are reflected in their expectations for faculty member productivity (that is, publication record or funding support), teaching loads and departmental responsibilities. Finally, many chemical biologists hold appointments in multiple departments, each of which applies its own set of standards. These factors, among others, contribute to the difficulty of defining a balanced set of expectations for chemical biologists and their scholarly work. The biophysics community has faced many of these challenges during its evolution as a cross-departmental field, and open dialog between biophysicists and chemical biologists may foster similar success in our community.

Because chemical biology centers on the use of molecular strategies to probe biological systems, new investigators initiate research programs by framing an important biological question and devising innovative ways to yield answers. This process depends on the background of the investigator. Biologists often continue projects that they started as postdoctoral fellows. In contrast, chemists are generally expected to set off in different research directions. Because many chemical biologists reside in chemistry departments, this implicit requirement for altering course can be a significant challenge. First, frontier areas of chemical biology are competitive; subdisciplines can be crowded with investigators, many of whom have greater experience and resources. The creation of an experimental or theoretical system requires a time investment that may compromise the competitiveness of a young laboratory. Second, to make rapid progress in a new area, a chemical biology laboratory requires talented students and postdoctoral researchers who are conversant in both chemistry and biology. Although traditional departmental programs excel in educating chemists or biologists, most novice chemical biology researchers require a substantial training period before they can make interdisciplinary contributions. Though most chemical biology faculty members agree on the pedagogic value of educating specialists more broadly, investigators are constantly reminded that this training phase can be an impediment to laboratory research.

Funding scientific research remains an ongoing issue for the entire biomedical research community, but it is particularly challenging for new investigators in chemical biology. Recent reports have highlighted the downward trend in success rates for grant applications at the US National Institutes of Health (Science 307, 1023, 2005), especially among young researchers. Proposed cuts in training grants, which provide an important mechanism to fund students at the chemistry-biology interface (Science 310, 1601, 2005), further jeopardize the ability of chemical biologists to launch and sustain their programs. Fortunately, almost everyone agrees that funding of new investigators is a major priority, and new funding mechanisms are appearing (http://grants.nih.gov/grants/new investigators/index.htm). Despite these encouraging signs, many chemical biologists still face challenges getting their proposals funded because of difficulties in finding an appropriate niche within funding agencies. Even though many potential scientific review groups exist, not all have the breadth of expertise necessary to assess chemical biology proposals.

Scientific conferences and colleagues at other institutions provide a valuable support network for chemical biologists, particularly during the early stages of their careers. Universities should work with allied organizations to enhance regional communities of chemical biologists. Programs such as the Frontiers of Science Discussion Groups of the New York Academy of Sciences (http://www.nyas.org/programs/frontiers.asp) or the annual Yale Chemical Biology Symposium (http://www.yale.edu/chemicalbiology/) may serve as excellent models for other regional activities.

In addition to these local networks, supportive mentorship is essential at researchers' home institutions. For the fortunate scientists at 'hotbed' institutions in chemical biology, the road may be much easier. However, most new investigators are in departments in which they may be one of a few chemical biologists, or perhaps the only one. We frequently hear the refrain "My colleagues don't understand what I do" from frustrated chemical biologists. Without active mentorship and support from colleagues, these scholars may become isolated, which compromises their ability to succeed. Institutions need to implement more effective strategies to cultivate young talent in frontier areas. Although young scholars obviously benefit from good mentorship, institutions also reap rewards through sensible stewardship of financial and human resources.

As universities and research institutes welcome new chemical biologists, they must accept greater responsibility for their integration into and success within current academic structures. Specifically, institutions should start by recognizing the unique challenges inherent in chemical biology research, establishing more effective mentoring strategies and developing consistent standards for the evaluation of chemical biologists. The emergence of chemical biology as a distinct discipline offers an unusual opportunity for scientists and administrators to provide innovative solutions to these challenging issues. 